\title{
A Novel Simple Convoluted Geometry to Design Frequency Selective Surfaces for Applications at ISM and UNII Bands
}

\author{
Vitor Fernandes Barros, Francisco Carlos G. da Silva Segundo, Antonio Luiz P. S. Campos, Sandro \\ Gonçalves da Silva \\ Federal University of Rio Grande do Norte, Natal, RN, Brazil e-mails: vfb3916@yahoo.com.br; \\ segundoengeletrica@gmail.com; antonio.luiz@pq.cnpq.br; sgsee@ufrnet.br \\ Alfredo Gomes Neto \\ Federal Institute of Paraíba, João Pessoa, PB, Brazil e-mails: alfredogomesjpa@gmail.com
}

\begin{abstract}
In this paper, we use a novel convoluted geometry to design a dual-band FSS with angular stability and independent polarization operation. The geometry allows the design of a dualband FSS to block ISM and UNII radio bands. We performed a parametric study with the commercial software ANSYS HFSS. A prototype of our proposed FSS design was built and its transmission characteristics were measured and compared with simulated results. We observed good agreement between numerical and experimental results. The proposed FSS realized a dual-band response at the required bands and it had angular stability and polarization independence.
\end{abstract}

Index Terms - FSS, Novel Convoluted Geometry, Dual-band Response, ISM, UNII.

\section{INTRODUCTION}

Frequency Selective Surface (FSS) are often constructed by a periodic array of conducting or aperture elements deposited in a dielectric layer. The FSS acts as a filter, which rejects some frequencies. The frequency response is dependent to incidence angle, on the polarization of the incident waves, planar geometry of the elements, the spacing between these elements within the array, and the thickness and permittivity of the substrate [1]. FSS are widely used in different applications such as spatial filters, radomes, absorbers, reducing radar cross section (RCS), and improving antennas' parameters [2 - 6].

In past years, an increase has been observed in the use of wireless communication systems, especially in unlicensed bands, such as ISM (Industrial, Scientific and Medical) and UNII (Unlicensed National Information Infrastructure) bands. Such increase by concurrent systems has worsened the interference problem, which not only affects the system performance, but also compromises its own transmission [7]. The Federal Communications Commission (FCC) established that ISM applications could occupy three bands. The first starts at $902 \mathrm{MHz}$ and finishes at $928 \mathrm{MHz}$, second band between 2.4 to $2.5 \mathrm{GHz}$ and the last one of 5.725 to $5.875 \mathrm{GHz}$. For UNII, the FCC established it at 5.15 to $5.35 \mathrm{GHz}$ and 5.725 to $5.825 \mathrm{GHz}$.

An eventual solution to this problem is to use a FSS that blocks this interference frequency between 
different systems. The FSS can be placed in walls following a wide variety of arrays, deliberately filtering out unwanted noise, while allowing certain radio services to pass [7]. In [8], the authors used convoluted elements to project FSS wallpaper to block unlicensed frequency range of $2.4 \mathrm{GHz}$, an ISM radio band. In [9], the authors enhance their previous work and study the stability for both TE and TM polarized waves not only for normal incident wave but also for oblique incidence angles. In [10], the authors showed a project of cascading FSS to block the second and third ISM bands and UNII band. In [11], the authors proposed a compact FSS to reject $5 \mathrm{GHz}$ band with angular stability for TE and TM modes of polarization. None of those projects blocked all ISM bands and UNII bands with angular stability and independence of polarization.

In [12], the authors used a fourth-order modified-Hilbert curve to design a single layer FSS for shielding ISM and UNII simultaneously with stable performance for both vertical and horizontal polarization under incident angles of $0^{\circ}$ through $60^{\circ}$.

In this project we propose an improved array where we used a single layer FSS to block all ISM and UNII bands and a stability study of polarization wave and incident angle was performed, in a much simpler design compared to the [12].

\section{FSS DESIGN}

In the FSS design, a convoluted geometry element was used. In [13], the authors introduced the word convoluted as a general term to describe geometries of unit cells in FSS arrays where complex conductor or slot structures twist, turn and in some cases interweave. It is generally known that convoluted elements produce narrow-bands and can be used at low frequencies due to their greater electrical length [14].

In a first step, we initially designed a four-legged loaded FSS [15] with an arm width of $0.5 \mathrm{~mm}$ and a length of $9 \mathrm{~mm}$, as seen in Fig.1(a), along with its dimensions. The white area in Fig. 1(a) represents apertures, while the black area is conducting patch. The aperture was changed until a value of $3.5 \mathrm{~mm}$ with the aim of resonance frequency being in the band comprising between 5 and $6 \mathrm{GHz}$. The next step was to insert a branch in the initial geometry as shown in Fig.1(b).

Fig. 2 illustrates the FSS. The spacing between each branch was $0.5 \mathrm{~mm}$. For the development of this work, we used the commercial software ANSYS HFSS to perform the numerical analysis of the transmission properties of the FSS. In the experimental array, we used a low cost dielectric FR-4 substrate with electrical constant permittivity $\left(\varepsilon_{r}\right)$ equal to 4.4 , thickness of $1.6 \mathrm{~mm}$ and a loss tangent equal to 0.02 . The periodicity in the $x$ and $y$ directions, was $20 \mathrm{~mm}$.

Fig. 3 shows the measured transmission coefficient for a normal incident wave. For each of the five branch levels (FSS 1 to 5) a new reject band appears. The FSS 5 realizes seven rejection bands. In FSS 2 we can see two rejection bands that block all ISM and UNII radio bands. We can see that more stop band means lower attenuation levels. This is because the irradiated energy is distributed in several bands, as a spectral scatter. In such way, more bands we add, lower is the attenuation levels. 
FSS 1

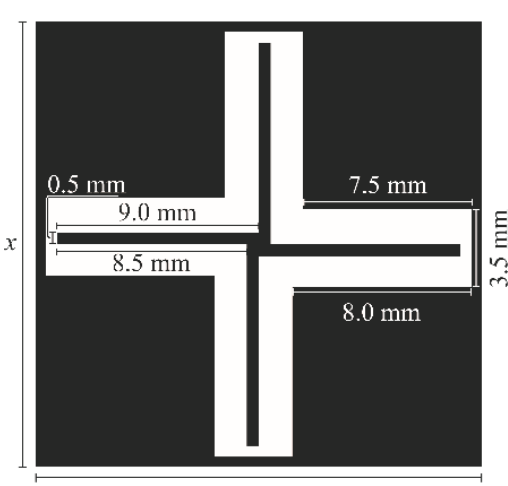

(a)
FSS 2

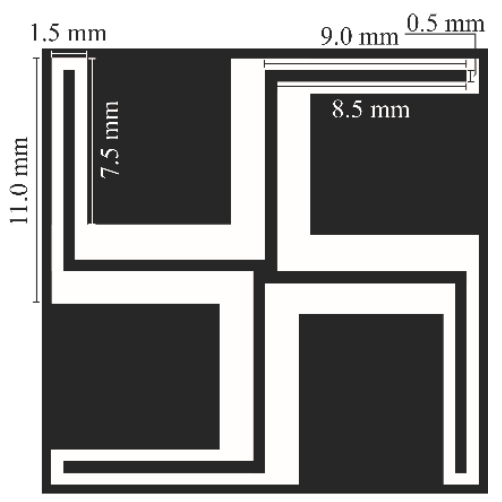

(b)

Fig. 1. Novel convoluted geometry: (a) Level 1 and (b) Level 2. FSS 3
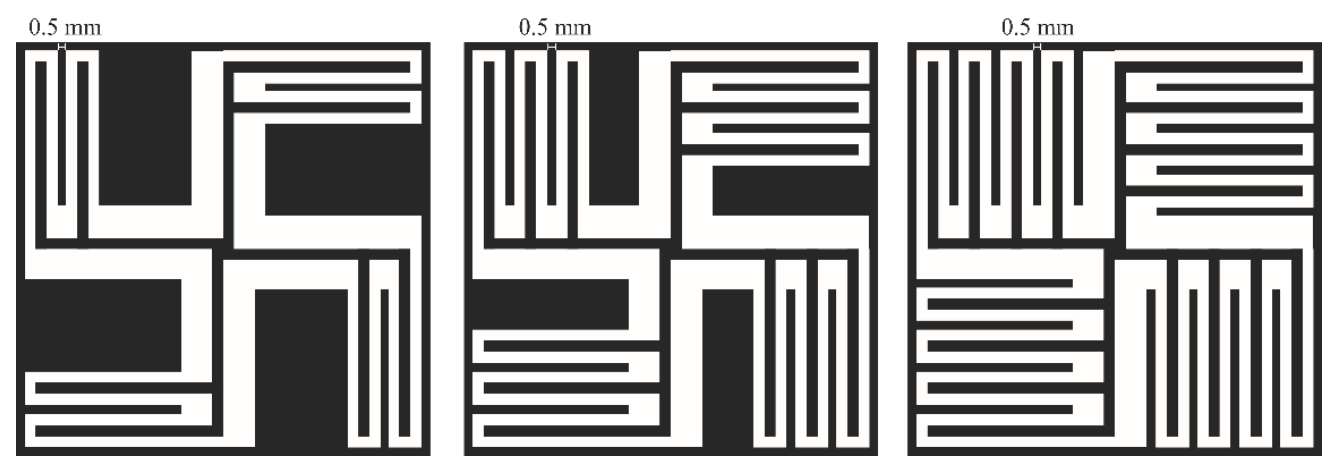

Fig. 2. Novel convoluted geometry levels 3 , 4, and 5

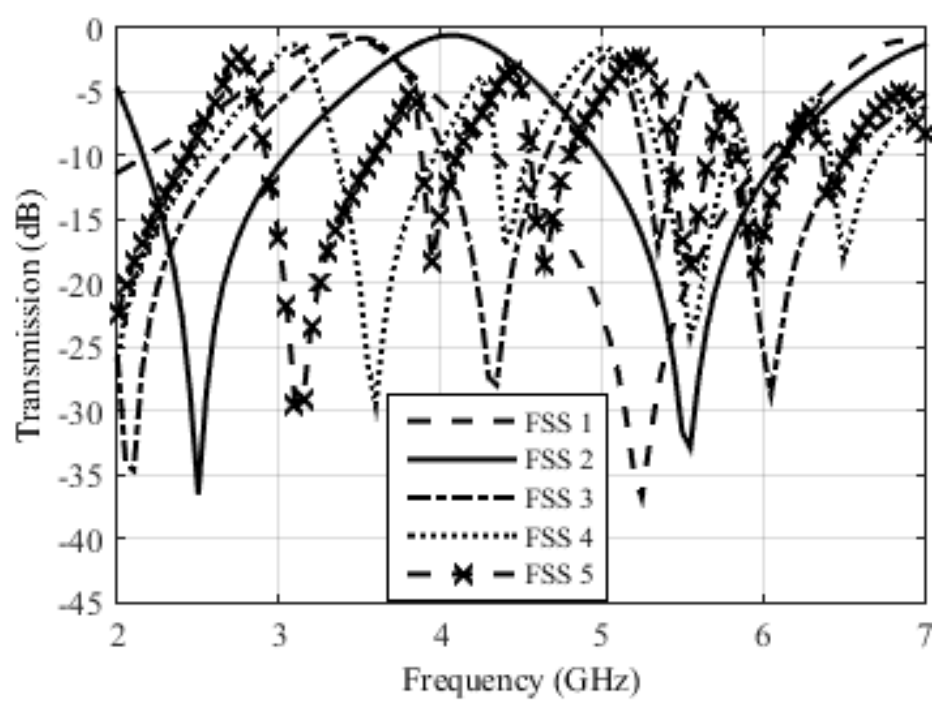

Fig. 3. The frequency response of FSS levels 1, 2, 3, 4, and 5.

In FSS 2, a study of the influence of the branch length as shown in Fig. 4 was developed. The length of the branch was adopted from $0 \mathrm{~mm}$ to $9 \mathrm{~mm}$ with the pitch of $1 \mathrm{~mm}$. 

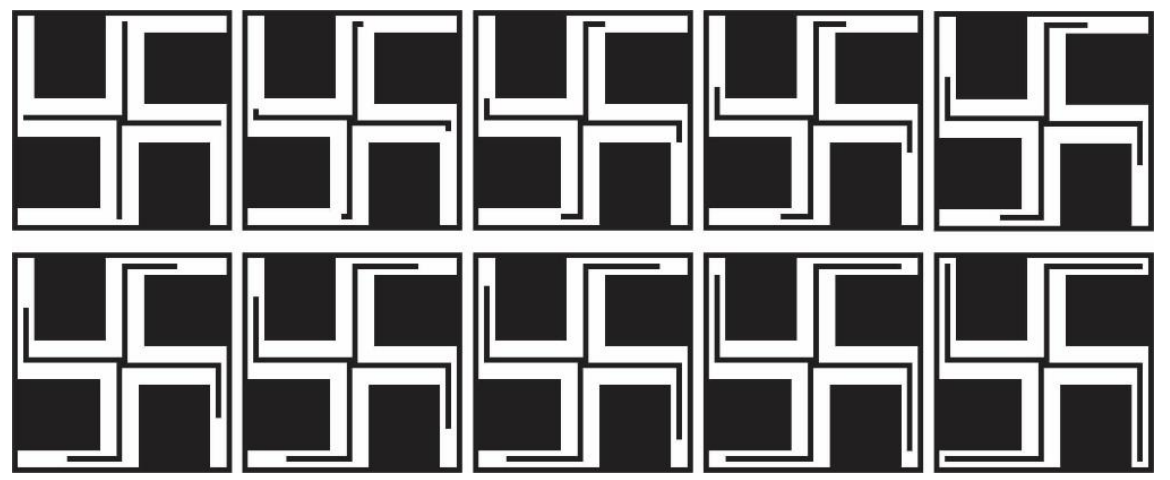

Fig. 4. Branch length variation of FSS 2.

Fig. 5 shows the simulated transmission coefficient for a normal incident wave in simulated cases shown in Fig. 4 for branch length changing from 0 to $4 \mathrm{~mm}$. Fig. 6 shows the transmission coefficient for branch length from 5 to $9 \mathrm{~mm}$.

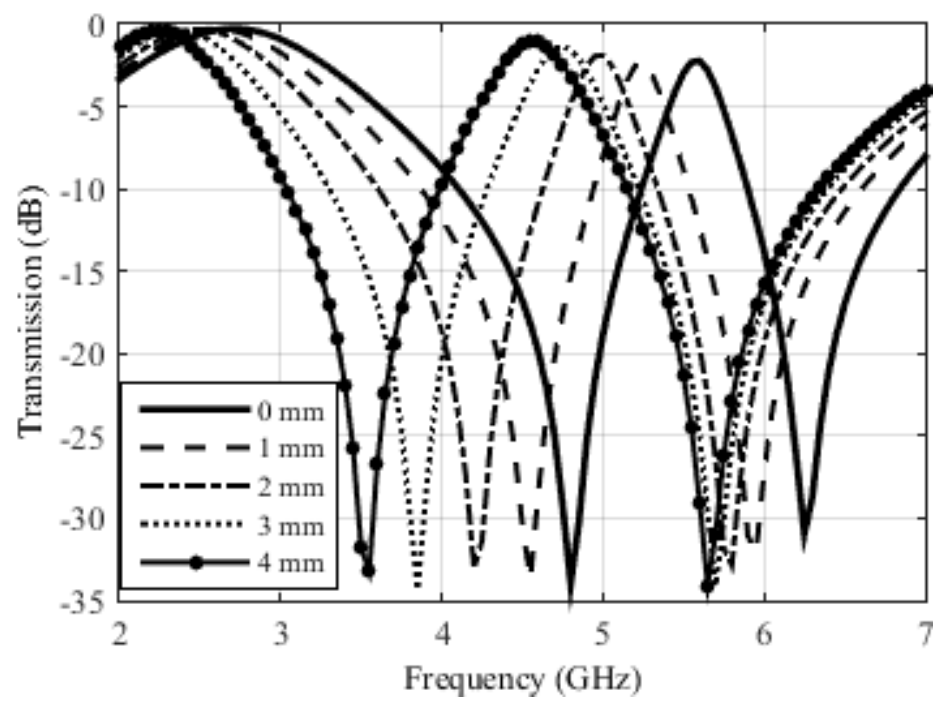

Fig. 5. Simulated frequency response for branch length from 0 to $4 \mathrm{~mm}$.

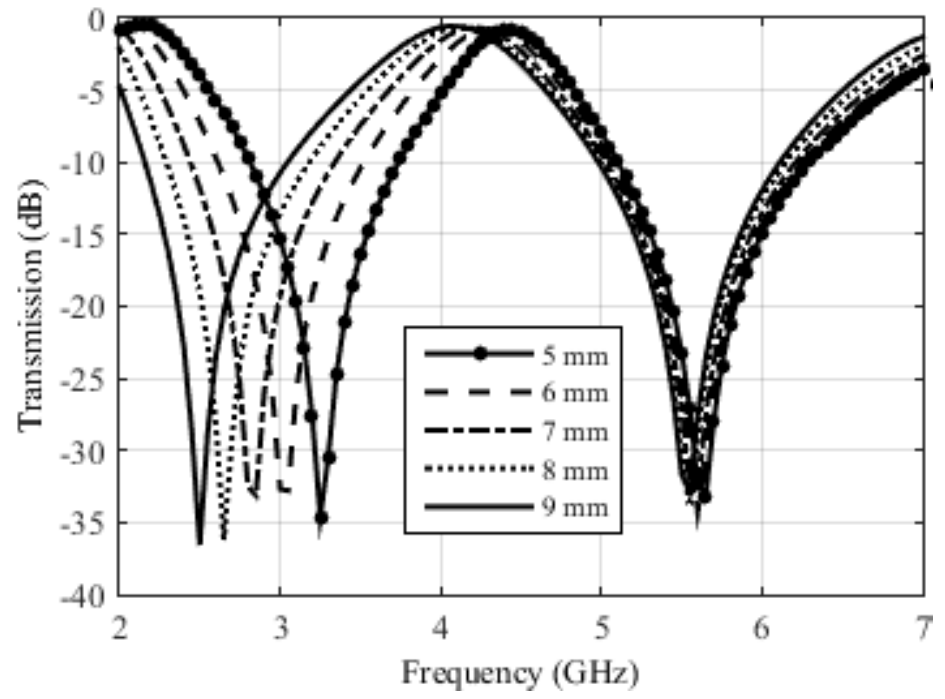

Fig. 6. Simulated frequency response for branch length from 5 to $9 \mathrm{~mm}$. 
In Fig. 5 and 6 we can observe that a changing of the branch length directly affects the first rejection band reducing its resonance frequency while the second band from $4 \mathrm{~mm}$ is little affected. The increase in branch length causes a distance between the rejection bands. Increasing the branch length means an increase in the electrical length and a decrease in the first resonance frequency and as the second band is not affected, the distance between resonances increases.

As shown in Fig. 7 a current distribution at each of the resonance frequency for FSS 2 with branch of $0 \mathrm{~mm}, 4 \mathrm{~mm}$ and $9 \mathrm{~mm}$. We can observe that the second resonance presents low or none variation in current distribution because of the modification of the branch length, while for the first resonance we can observe a significant changing in current distribution because of the modification of the branch length.

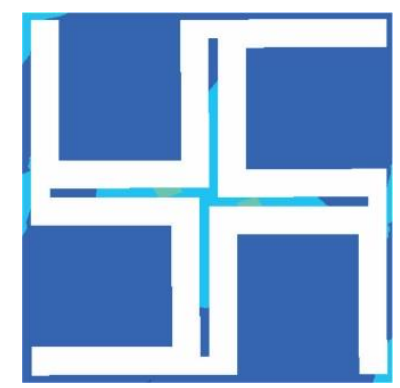

(a)

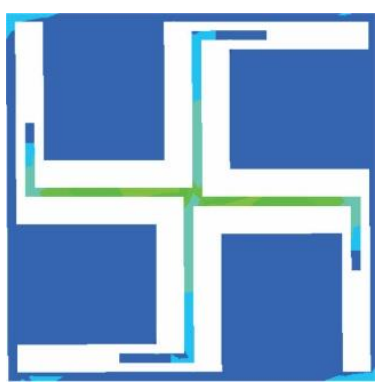

(c)

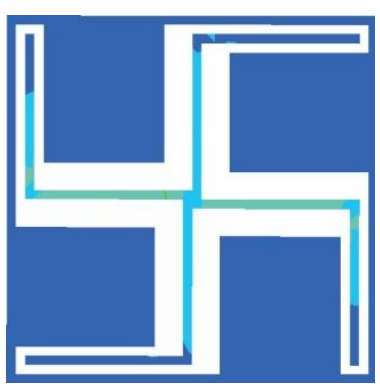

(e)

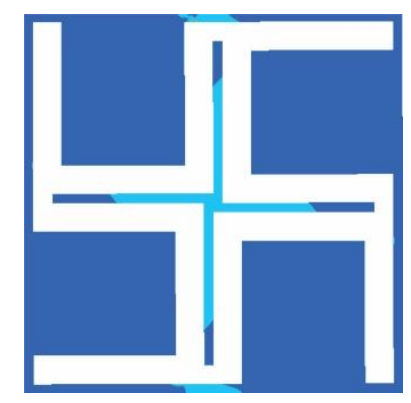

(b)

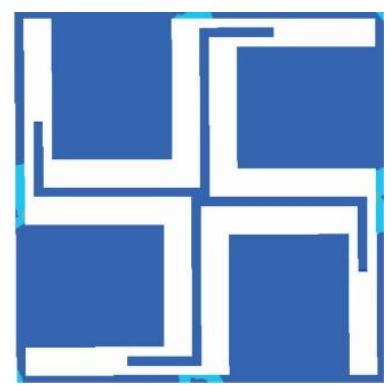

(d)

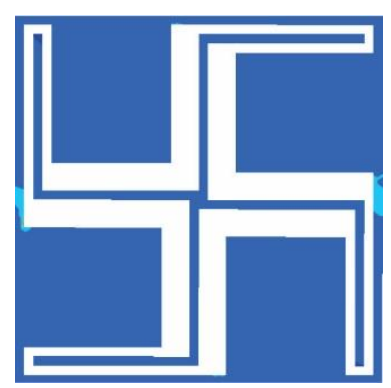

(f)

Fig. 7. Current distribution: (a) Resonant frequency of $4.8 \mathrm{GHz}$ and (b) Resonant frequency of $6.25 \mathrm{GHz}$ with length of branch of $0 \mathrm{~mm}$; (c) Resonant frequency of $3.55 \mathrm{GHz}$ and (d) Resonant frequency of $5.65 \mathrm{GHz}$ with length of branch of 4 $\mathrm{mm}$; (e) Resonant frequency of $2.5 \mathrm{GHz}$ and (f) Resonant frequency of $5.55 \mathrm{GHz}$ with length of branch of $9 \mathrm{~mm}$.

In Fig. 8 we can see the FSS used to study the tape width in frequency response of FSS. The FSS designed with a strip width of $0.5 \mathrm{~mm}$ to $2.5 \mathrm{~mm}$ with a step of $0.5 \mathrm{~mm}$. 

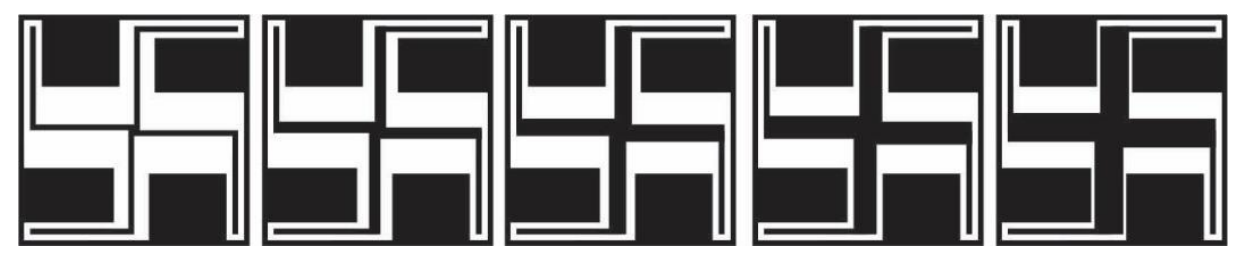

Fig. 8. Strip width variation of FSS 2.

In Fig. 9 is shown the frequency response of FSS as a function of the strip width. As observed in Fig. 9, width influence causes a variation in first resonant frequency. Second band is not affected. So when increase the width tape, the resonant frequencies tend to come together.

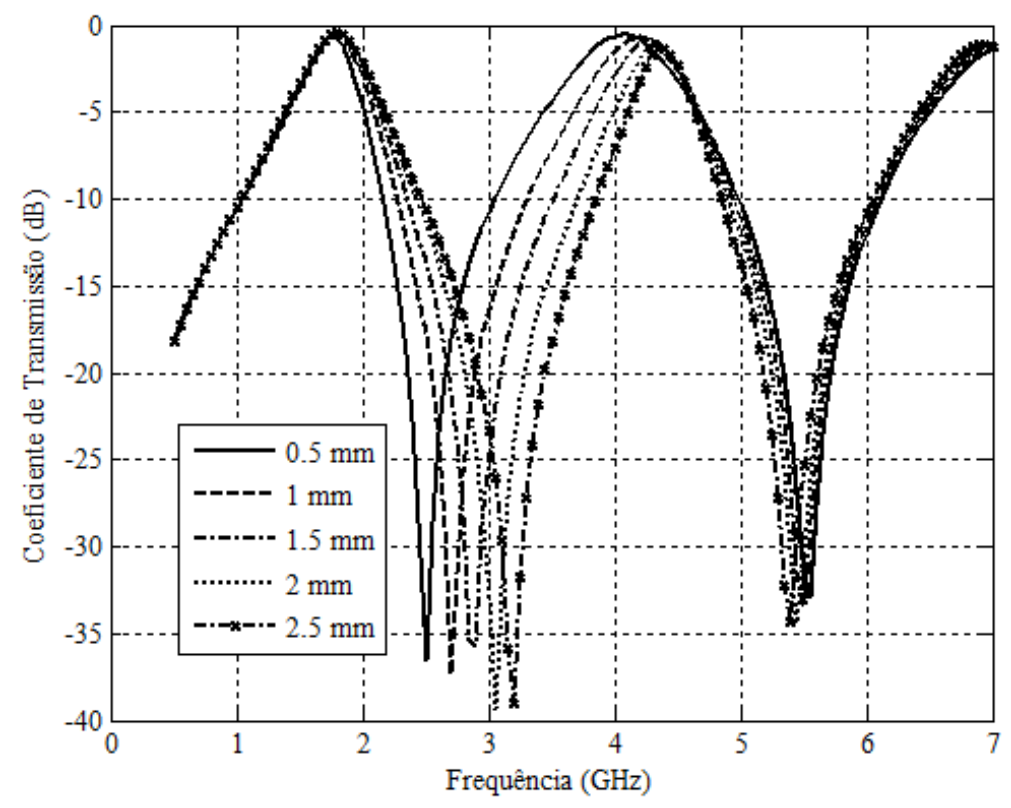

Fig. 9. Simulated frequency response for strip width from 0.5 to $2.5 \mathrm{~mm}$.

\section{RESULTS AND DISCUSSIONS}

By following, after this parametric analysis, we chose the FSS 2 with branch lengths of $9 \mathrm{~mm}$ to design the desired FSS and we performed an angular stability analysis. Fig. 10 shows the frequency response of our proposed FSS design for different incident angles and for vertical polarization. Note that there is no degradation in resonance frequency and bandwidth, which shows that the designed FSS has angular stability.

As the results in Fig. 11 shows, for horizontal polarization, only incident angles at the $2.5 \mathrm{GHz}$ band have angular stability. Also, on the other band, there is a reduction of the bandwidth, but it still contains the UNII band. This behavior can occur because of some adjust in simulations. We fixed a mesh four times bigger than conventional mesh used and it still occurs. 


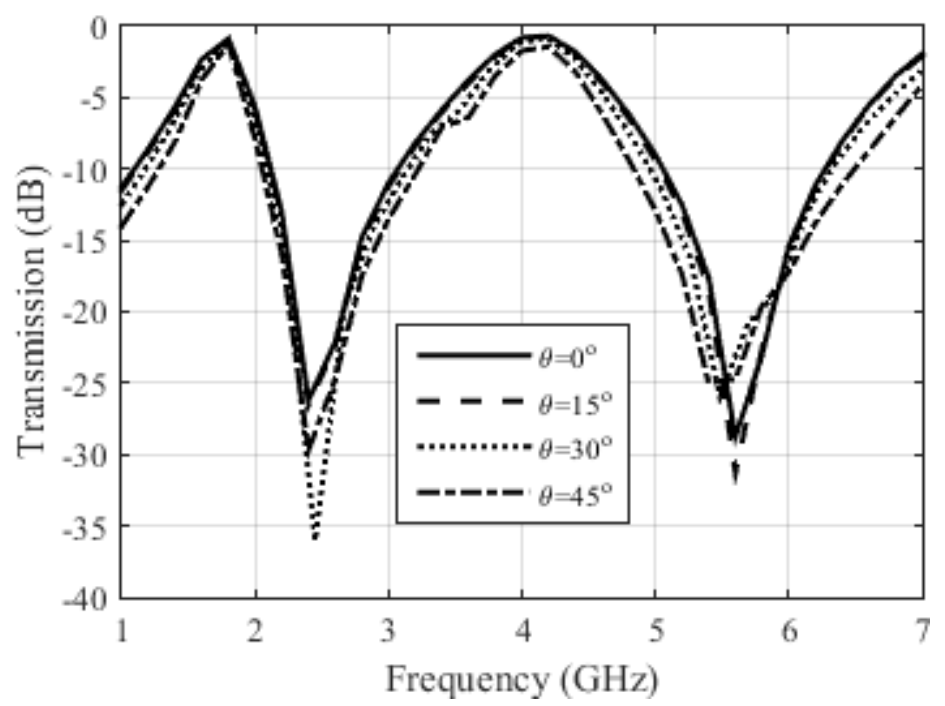

Fig. 10. Simulated frequency response of our proposed FSS design for different angles of incidence in vertical polarization.

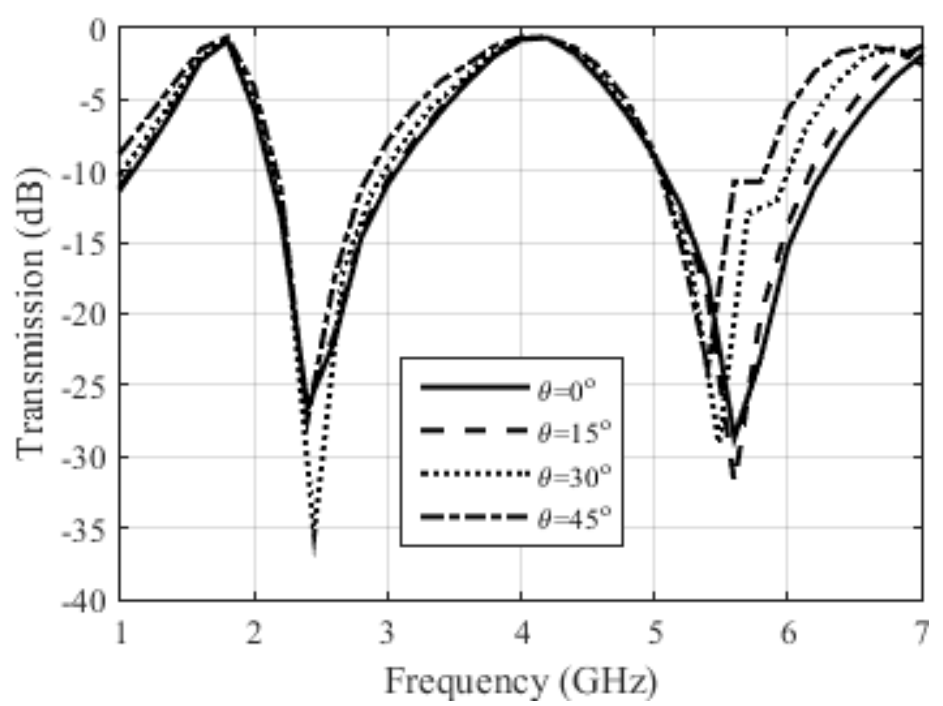

Fig. 11. Simulated frequency response of our proposed FSS design for different angles of incidence in horizontal polarization.

After the simulations, we built a prototype to validate the simulated results. The built prototype is shown in Fig. 12.

The transmission characteristics of the structures were measured using a network analyzer from Agilent, model N5230A, and two wideband Vivaldi horn antennas. We analyzed the results obtained in the frequency range from $2.0 \mathrm{GHz}$ to $7.0 \mathrm{GHz}$, as can be seen in Fig. 13. 


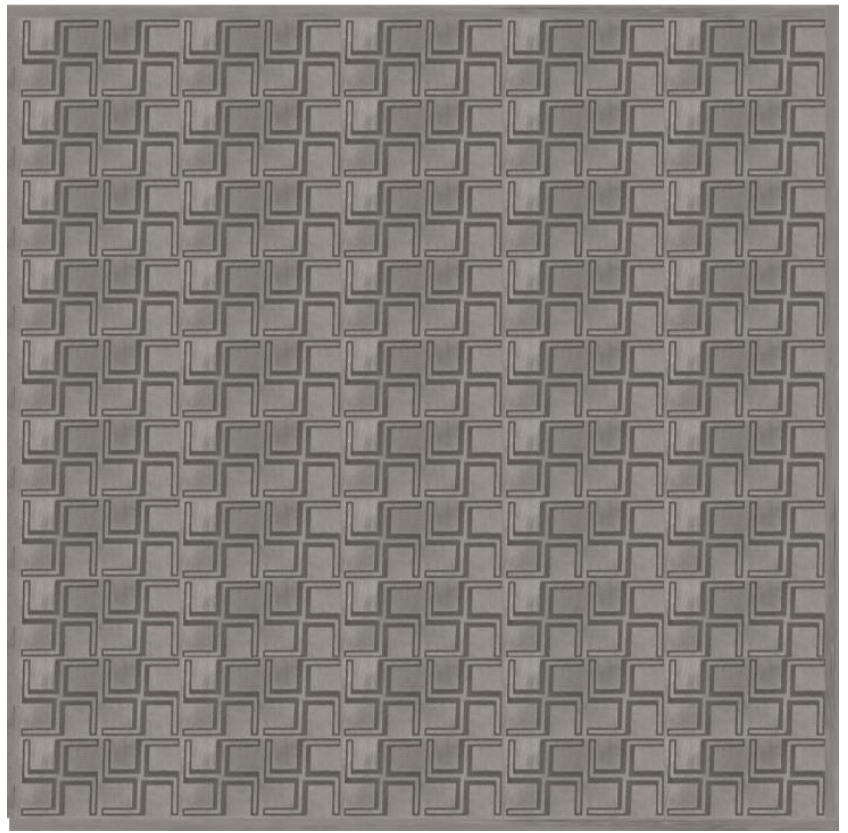

Fig. 12. Fabricated prototype.

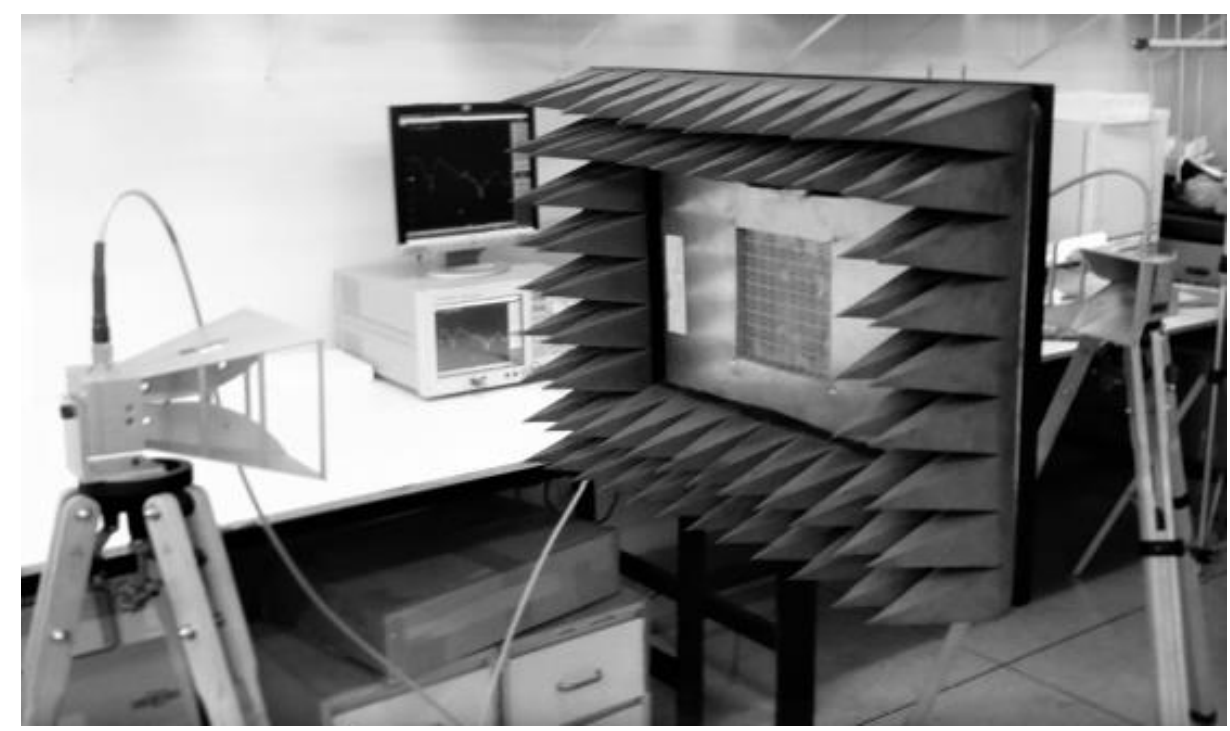

Fig. 13. Measurement setup.

The Fig. 14 presents a good agreement when comparing the simulated and measured results for normal incidence, presenting a first bandwidth equal to $880 \mathrm{MHz}(2.28-3.16 \mathrm{GHz})$ and a second bandwidth equal to $1300 \mathrm{MHz}(5.14-6.44 \mathrm{GHz})$, both appropriate to operate in the entire ISM and UNII bands. 


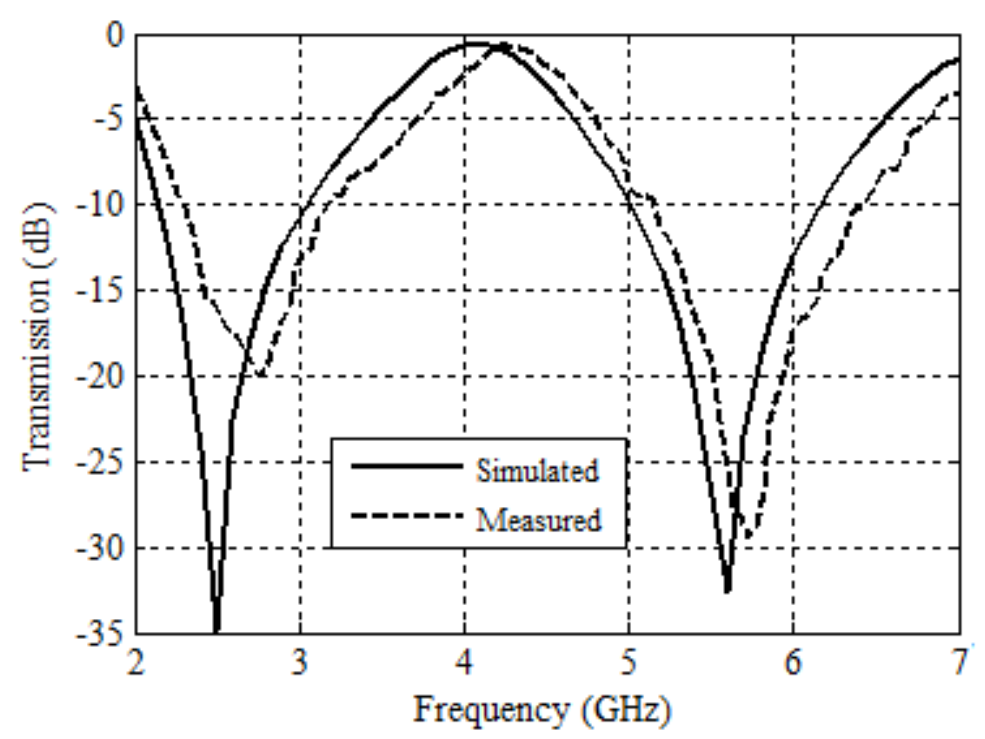

Fig. 14. Comparison between measured and simulated results for normal incidence.

Fig. 15 shows the frequency response of our proposed FSS design for different angles of incidence and vertical polarization. We can note that there is no degradation in resonance frequency and bandwidth. This fact shows that the designed FSS has angular stability.

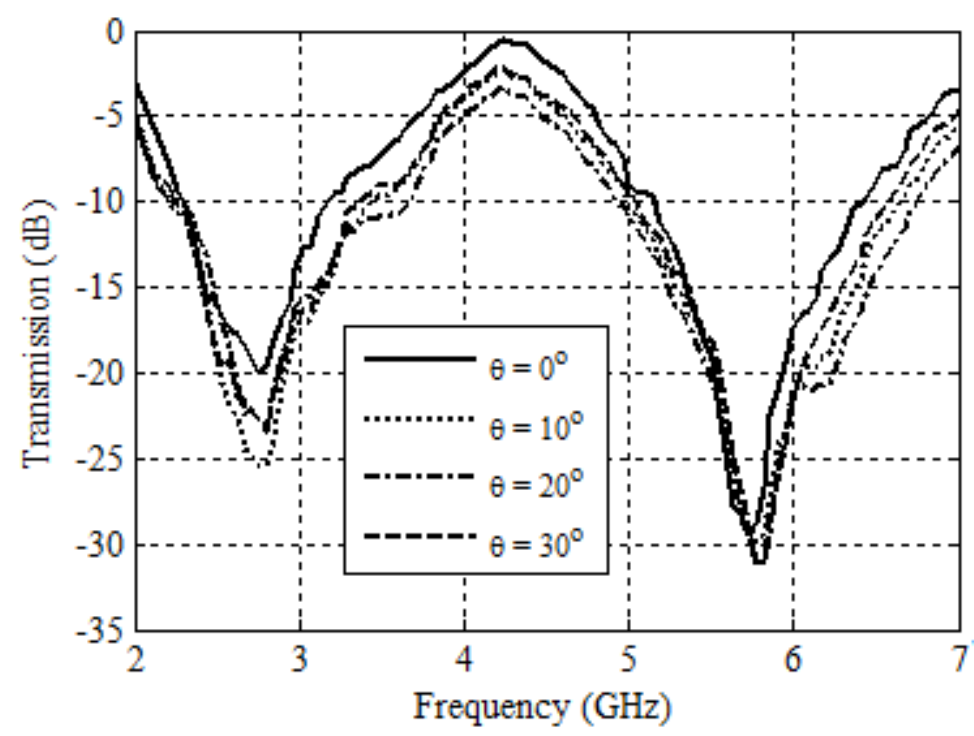

Fig. 15. Measured frequency response of our proposed FSS design for different angles of incidence and vertical polarization.

Fig. 16 illustrates the frequency response of our proposed FSS design for different angles of incidence for horizontal polarization. Again, there is no degradation in bandwidths. This fact shows that the designed FSS has polarization independence. Different from the simulated results, experimental results showed an angular stability for both polarizations. 


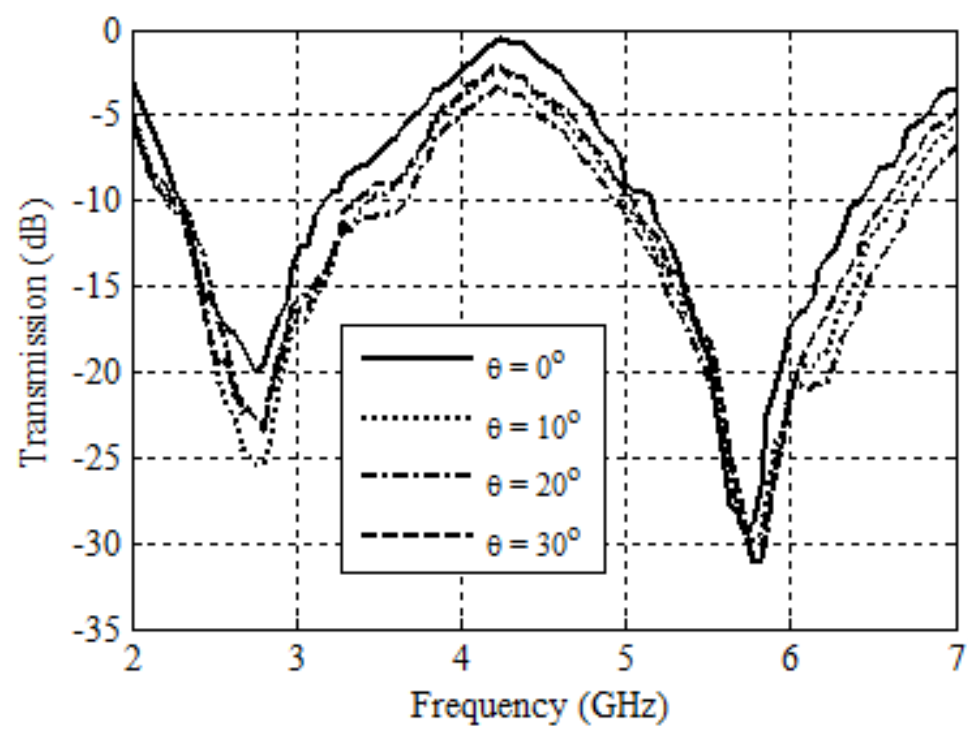

Fig. 16. Measured frequency response of designed FSS for different angles of incidence and horizontal polarization.

\section{CONCLUSIONS}

In this paper, we used a novel convoluted geometry element to design a FSS with two bands of resonance, rejecting all ISM and UNII bands. The FSS can be used to limit a radio link or to reduce interference, allowing systems to continue to operate effectively in the presence of interfering signals. By building a prototype of a new FSS design and measuring its transmission characteristic, the results showed that such design provides attenuation levels ranging from 10 to $20 \mathrm{~dB}$ at ISM band and from 10 to $30 \mathrm{~dB}$ at UNII band. Additionally, our FSS design keeps the simplicity feature in the array, since it uses only one dielectric layer, that proved to be compact and lightweight, also presenting angular stability and independence of polarization.

\section{ACKNOWLEDGMENT}

The authors express their sincere thanks to the National Council of Research and Development CNPq for supporting the research work over the project 303693/2014-2.

\section{REFERENCES}

[1] Wu, T. K., "Frequency selective surface and grid array", Jonh Wiley \& Sons, New York, E.U.A., 1995.

[2] Munk, B. A. Frequency selective surfaces: Theory and design, New York, Wiley, New York, NY, 2000.

[3] Campos, A. L. P. S., Superfícies Seletivas em Frequência - Análise e Projeto (in portuguese), Editora do IFRN, 2010.

[4] Seman, F. C. and Cahill, R., "Frequency Selective Surfaces Based Planar Microwave Absorber", PIERS Proceedings, Kuala Lumpur, Malaysia, (2012), $906-909$.

[5] Turpin, J. P., Sieber, P. E., and Werner, D. H., “Absorbing FSS ground plane for reduced-Radar Cross Section of conformal antennas", Proceedings of Antennas and Propagation Symposium, Orlando, United States, (2013), 464 - 465.

[6] Almeida Filho, V. A. and Campos, A. L. P. S., "Performance optimization of microstrip antenna array using frequency selective surfaces", Journal of Microwaves, Optoelectronics and Electromagnetic Applications, Vol. 13, No. 1, (2014), 31 - 46.

[7] Sung, G. H., Sowerby, K. W., Neve, M. J., and Williamson, A. G., "A frequency-selective wall for interference reduction in wireless indoor environments", IEEE Antennas and Propagation Magazine, Vol. 48, No. 5, (2006), 29 - 37.

[8] Alkayyali, H. and Qasem, N., "Convoluted Frequency Selective Surface Wallpaper to Block the Industrial, Scientific, and Medical Radio Bands Inside Buildings". American Academic \& Scholarly Research Journal. Vol. 5, No. 3, (2013).

[9] Kartal, M., Pinar, S. K., Doken, B., and Gungor, I., "A new narrow band frequency selective surface geometry design at unlicensed 2.4 GHz ISM Band”. Microwave and Optical Technology Letters, Vol. 55, No 12, (2013), 2986-2990.

[10] da Silva Segundo, F. C. G. and Campos, A. L. P. S., "Compact Frequency Selective Surface with Dual Band Response for WLAN Application". Microwave and Optical Technology Letters, Vol 57, No 2, (2015), 265 - 268.

[11] Rajesh Natarajan, Malathi Kanagasabai, Sanjay Baisakhiya, Ramprabhu Sivasamy, Sandeepkumar Palaniswamy, and Jayaram Kizhekke Pakkathillam, “A Compact Frequency Selective Surface With Stable Response for WLAN Application”. IEEE Antennas and Wireless Propagation Letters, Vol 12, (2013), 718-720. 
[12] Hussain, T., Yang, C., Cao, Q., \& Majid, I. "Electromagnetic shielding for WLAN using modified-Hilbert fractals". In Computational Electromagnetics (ICCEM), 2016 IEEE International Conference on, (2016), (pp. 132-134).

[13] E. A. Parker and A. N. A. El Sheikh, "Convoluted array elements and reduced size unit cells for frequency-selective surfaces," Proc. Inst. Elect. Eng. Microw., Antennas Propag., vol. 138, pt. H, pp. 19-22, Feb. 1991.

[14] Chuprin, A. D., Parker, E. A. and Batchelor, J. C, "Convoluted double square: single layer FSS with close band spacings," Electronics Letters, Vol 36, No 22, 1830-1831, 2000.

[15] Larry Epp, Chi Chan and Raj Mittra, "The study of FSS surfaces with varying surface impedance and lumped elements," Abstracts of the 1989 IEEE AP-S International Symposium, San Jose, CA, vol. 2, pp. 1056-1059, June 1989. 